\title{
Designing Three-Dimensional Flat Bands in Nodal-Line Semimetals
}

\author{
Alexander Lau $\odot,{ }^{1, *}$ Timo Hyart, ${ }^{1,2, *}$ Carmine Autieri@ ${ }^{1}$ Anffany Chen, ${ }^{3,4}$ and Dmitry I. Pikulin ${ }^{5,6}$ \\ ${ }^{1}$ International Research Centre MagTop, Institute of Physics, Polish Academy of Sciences, \\ Al. Lotników 32/46, 02-668 Warsaw, Poland \\ ${ }^{2}$ Department of Applied Physics, Aalto University, 00076 Aalto, Espoo, Finland \\ ${ }^{3}$ Department of Physics and Astronomy, University of British Columbia, \\ Vancouver, British Columbia, Canada V6T $1 Z 1$ \\ ${ }^{4}$ Quantum Matter Institute, University of British Columbia, \\ Vancouver, British Columbia, Canada V6T $1 Z 4$ \\ ${ }^{5}$ Station Q, Microsoft Corporation, Santa Barbara, California 93106-6105, USA \\ ${ }^{6}$ Microsoft Quantum, Redmond, Washington 98052, USA
}

(Received 5 November 2020; revised 25 March 2021; accepted 18 May 2021; published 22 July 2021)

Electrons with large kinetic energy have a superconducting instability for infinitesimal attractive interactions. Quenching the kinetic energy and creating a flat band renders an infinitesimal repulsive interaction the relevant perturbation. Thus, flat-band systems are an ideal platform to study the competition of superconductivity and magnetism and their possible coexistence. Recent advances in the field of twisted bilayer graphene highlight this in the context of two-dimensional materials. Two dimensions, however, put severe restrictions on the stability of the low-temperature phases due to enhanced fluctuations. Only threedimensional flat bands can solve the conundrum of combining the exotic flat-band phases with stable order existing at high temperatures. Here, we present a way to generate such flat bands through strain engineering in topological nodal-line semimetals. We present analytical and numerical evidence for this scenario and study the competition of the arising superconducting and magnetic orders as a function of externally controlled parameters. We show that the order parameter is rigid because the three-dimensional quantum geometry of the Bloch wave functions leads to a large superfluid stiffness in all three directions. Using density-functional theory and numerical tight-binding calculations, we further apply our theory to strained rhombohedral graphite and $\mathrm{CaAgP}$ materials.

DOI: 10.1103/PhysRevX.11.031017

Subject Areas: Condensed Matter Physics, Magnetism Superconductivity

\section{INTRODUCTION}

The study of correlated many-particle states in flat-band systems goes back to the consideration of few-particle nuclear-physics systems in the 1960s when Belyaev demonstrated that, in the presence of degenerate single-particle states, interactions can lead to a pairing gap increasing linearly with the interaction strength [1]. Since then, there has been a fruitful exchange of ideas between the nuclearphysics and the condensed-matter communities exploring analogies between nuclear-physics systems and ultrasmall superconducting grains [2-4]. Recently, two-dimensional (2D) flat bands have provided new ground for exotic states of condensed matter [5-9]. In particular, the advances in the

\footnotetext{
*These two authors contributed equally.
}

Published by the American Physical Society under the terms of the Creative Commons Attribution 4.0 International license. Further distribution of this work must maintain attribution to the author(s) and the published article's title, journal citation, and DOI. fabrication of flat bands in twisted bilayer graphene have attracted a lot of attention due to the novel exotic phases becoming available experimentally [10-16]. Other setups and materials realizing 2D flat bands have been studied [17-19] but, to our knowledge, their three-dimensional (3D) counterparts $[20,21]$ have not been explored in realistic materials. In the present work, we carry this development to its logical end point by proposing a viable approach for realizing 3D flat-band systems.

We combine two actively studied ingredients to manifest a 3D flat band: nodal-line semimetals (NLSMs) [22] and strain engineering in topological semimetals [23]. NLSMs are materials where topologically protected band crossings form a line (nodal line) in the Brillouin zone, provided that certain symmetries are satisfied. Additionally, topologically protected drumhead surface states appear inside the region bounded by the projection of the nodal line onto the $2 \mathrm{D}$ surface Brillouin zone. The drumhead surface states exhibit a nearly flat dispersion if the material has approximate chiral symmetry, for example, due to a sublattice structure [7]. 
Strain engineering produces opportunities to generate Landau level-like flat bands in the absence of external magnetic fields [24-30] because the action of the strain near the Fermi surface resembles that of a magnetic field in the local area of the Brillouin zone. In the case of Weyl or Dirac semimetals, there are simply two Fermi pockets near the Fermi energy, and the action of strain near the pockets can be described as electromagnetic fields [31-33]. Here, we show that such properties of strain are more generic and can be applied to materials with nodal lines as well. For stationary strain, the resulting pseudomagnetic field then depends on the position along the nodal line. However, there is always one commonality in nodal systems insensitive to the strength and direction of the pseudomagnetic field - the zeroth pseudo-Landau level (PLL). Therefore, one would expect that the zeroth PLL forms a 3D flat band, while the higher PLLs are flat only in two dimensions and have a dispersion along the nodal line.

We confirm this intuitive argument above using numerical and analytical arguments. We show that the zeroth PLL indeed forms a 3D flat band, which evolves from the drumhead surface states of the NLSM, and we obtain wave functions thereof. Using these wave functions and assuming competition between magnetism and superconductivity, we obtain the phase diagram of the system as a function of the filling factor and the interaction strengths. We show that this system is a promising platform for studying intertwined phases [9,34], as it can be tuned in situ from the magnetic to the superconducting phase by controlling the magnitude of the strain.

Moreover, we go beyond the mean-field picture by analyzing the properties of the collective modes and argue that the emerging order is more stable in the $3 \mathrm{D}$ case than in the previously studied 2D cases. Importantly, we show that the system supports a hitherto unexplored 3D quantum geometry of the Bloch wave functions that leads to a large superfluid stiffness in all directions despite the flatness of the bands. The 3D quantum geometry is a nontrivial consequence of the momentum dependence of the direction of the pseudomagnetic field along the nodal line. Finally, we study the properties of 3D flat bands for the material examples of NLSMs belonging to the CaAgP material class and of rhombohedral graphite.

\section{NONINTERACTING MODEL}

\section{A. Two-band NLSM Hamiltonian and drumhead surface states}

A NLSM is a material for which the energy gap between two bands near the Fermi level closes along a line in the Brillouin zone [Fig. 1(a)]. The minimal model for a NLSM - believed to be applicable, for instance, to the $\mathrm{CaAgP}$ materials class (see below) and $\mathrm{Ca}_{3} \mathrm{P}_{2}$ [35-37]reads
$H(\mathbf{k})=\sigma^{z}\left(6 t-t_{1}-2 t \sum_{i=x, y, z} \cos k_{i}\right)+2 t_{2} \sigma^{x} \sin k_{z}$.

Here, $\sigma^{x, y}$ are Pauli matrices, and $t, t_{1}$, and $t_{2}$ are parameters determining the size of the nodal line and the Fermi velocity. Throughout this paper, the length scales are given in units of the lattice constant, and the momentum is measured in units of the inverse lattice constant. For $t_{1} \ll t$, this Hamiltonian has a gap closing along a circular path in the Brillouin zone-a nodal loop-given by $k_{z}=0$ and $\left(k_{x}, k_{y}\right)=\mathbf{Q}=Q(\cos \theta, \sin \theta)$, where $Q=\sqrt{t_{1} / t}$ and $\theta$ is the azimuthal angle. The crossing between the two bands is protected by the $x-y$ plane reflection symmetry. The bands are $\sigma^{z}$ eigenstates in the $k_{z}=0$ plane corresponding to reflection eigenvalues \pm 1 . Taking a different viewpoint, the nodal line can also be considered to be protected by the chiral symmetry $\sigma^{y}$ or the combination of time-reversal and inversion symmetries. For this Hamiltonian, the drumhead surface states, appearing at momenta $k<Q$, have zero energy because of chiral symmetry. In the limit of small nodal-line radii $Q$, the corresponding surface wave functions at momentum $\mathbf{k}=\mathbf{Q}+\mathbf{q}$ can be written as

$$
\Psi_{S, \pm}(\mathbf{Q}, \mathbf{q})=\frac{1}{\sqrt{2 L_{x} L_{y} l_{S} \sinh \frac{L}{l_{S}}}}\left(\begin{array}{c}
1 \\
\pm i
\end{array}\right) e^{i(\mathbf{Q}+\mathbf{q}) \cdot \mathbf{r}} e^{ \pm\left(z / l_{S}\right)}
$$

where $\mathbf{q}=q(\cos \theta, \sin \theta)$ and $q<0$ describes the deviation from the nodal line. Here, \pm correspond to top and bottom surfaces, $-L / 2<z<L / 2, l_{S}=-t_{2} /(t Q q)$ is the localization length of the drumhead surface states, and $L_{x}$ $\left(L_{y}\right)$ is the length of the system in the $x(y)$ direction along which we have applied periodic boundary conditions.

\section{B. Strain and pseudo-Landau levels}

A simple way to realize strain with a constant gradient of the strain field is by bending $[23,39]$, as shown in Fig. 1(b). The bend can be achieved by a proper choice of the growth substrate or by mechanical bending of the device. Such strain creates a displacement field $\mathbf{u}=(x z / R, 0,0)$ with corresponding strain tensor components $u_{11}=z / R$ and $u_{13}=u_{31}=x /(2 R)$. In the limit of small nodal-line radii, the strain induces a pseudomagnetic field (see Supplemental Material [38])

$$
\mathbf{B}_{5}=\frac{\hbar}{e l_{B}^{2}}(\sin \theta,-\cos \theta, 0)
$$

where $\mathbf{Q}=Q(\cos \theta, \sin \theta)$ is a momentum along the nodal line and $l_{B}=\sqrt{R Q}$ is the pseudomagnetic length. In this case, the analytical solution for the zeroth PLL wave function is 
(a)

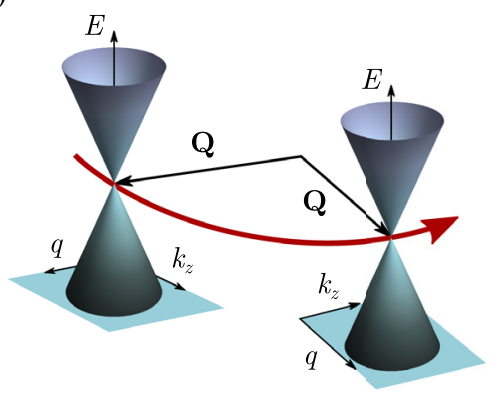

(d)

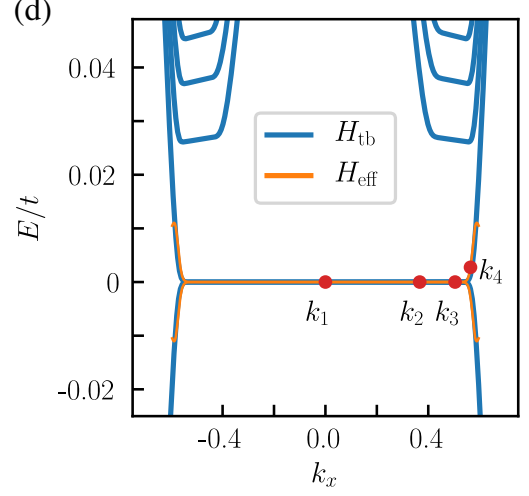

(b)

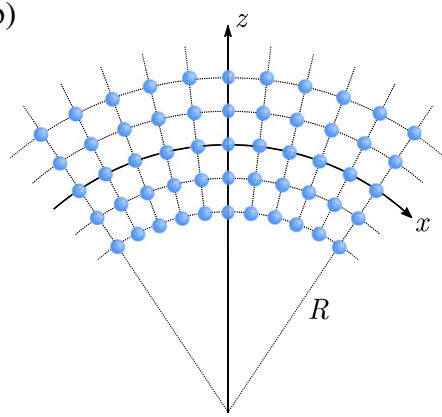

(c)

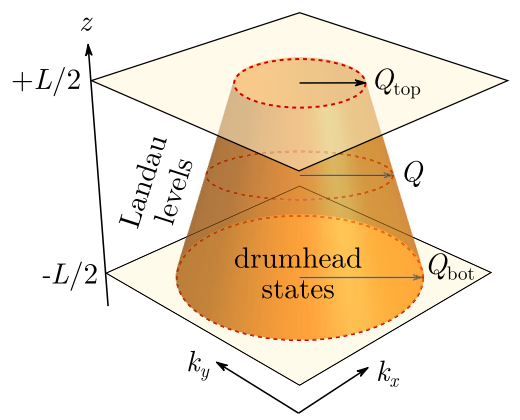

(e)

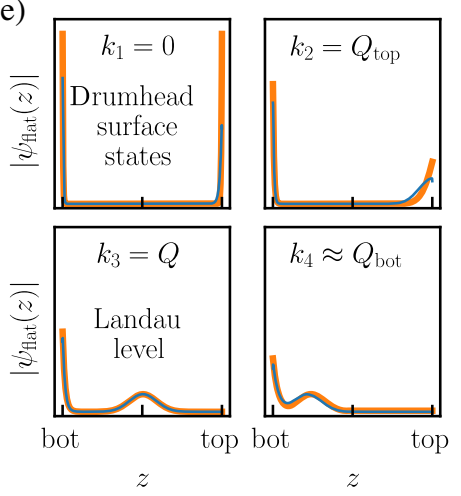

(f)

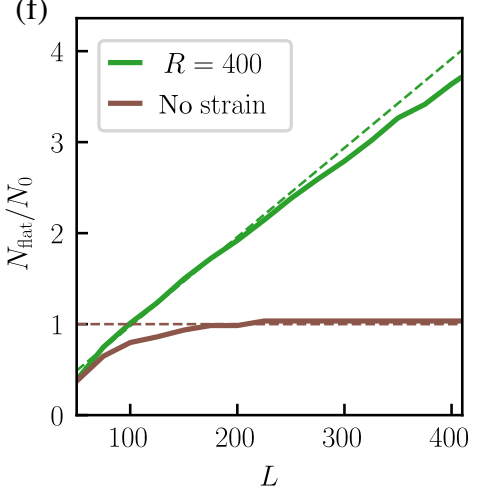

FIG. 1. (a) Schematic representation of a nodal-line semimetal band structure. Here, $\mathbf{Q}$ is a vector pointing to a momentum on the nodal line such that $|\mathbf{Q}|=Q$ is the radius of a circular nodal loop. A Dirac cone is formed with respect to the perpendicular momentum components $q$ and $k_{z}$. (b) Example of a strain profile leading to the formation of a 3D flat band. The strain can be created, e.g., by bending the sample or growing it on a cylindrical surface. Here, $R$ is the radius of the cylinder in the middle of the sample. (c) Bulkboundary correspondence of the zeroth PLL of the NLSM. Because of the strain, the radius of the nodal circle varies as a function of $z$, such that the radius of the momentum-space area of the drumhead surface states on the top surface $Q_{\text {top }}$ is different from the bottom surface $Q_{\text {bot }}$. Zeroth PLL bulk states appear in the momentum space region between $Q_{\text {top }}$ and $Q_{\text {bot }}$. (d) Spectrum of the model Hamiltonian $H_{\mathrm{tb}}$ [Eq. (1)] in the presence of strain [Eq. (2)] with parameters $t_{1}=0.25 t, t_{2}=0.8 t, L=1000$, and $R=8000$. For comparison, we also show the energies of the effective Hamiltonian $H_{\text {eff }}$ (orange bands) constructed from the analytical low-energy solutions (see Supplemental Material [38]). (e) Numerical (blue) and analytical (orange) wave functions of the zeroth PLL at different momenta $k_{x}=k_{i}(i=1,2,3,4)$ indicated in panel (d). The drumhead surface states appear at both surfaces for $\left|k_{x}\right|<Q_{\text {top }}, Q_{\text {bot }}$. At $\left|k_{x}\right|=Q_{\text {top }}$, the drumhead surface states from the top surface are deformed into the zeroth PLL bulk states and move towards the bottom surface with increasing $Q_{\text {top }}<\left|k_{x}\right|<Q_{\text {bot }}$. At $\left|k_{x}\right|=Q_{\text {bot }}$, the PLL bulk state hybridizes with the drumhead surface state at the bottom surface. (f) Degeneracy of the flat band as a function of the height $L$ of the sample. Numerically, the degeneracy is calculated by integrating the density of states over an energy window $\left(|E|<10^{-3} t\right)$. The model parameters $t_{1,2}$ are the same as in panel (d). Bold lines are the numerical results, and dashed lines correspond to the analytical formulas. In the presence of strain, the degeneracy grows linearly with $L$, demonstrating the existence of a 3D flat band with degeneracy $N_{\text {flat }}$ proportional to the volume of the sample (green dashed line). In the absence of strain, the degeneracy of the zero-energy drumhead surface states saturates with increasing $L$, demonstrating that it is proportional to the area of the surface of the sample, $N_{0}$.

$$
\Psi_{0}(\mathbf{Q}, \mathbf{q})=\frac{1}{\sqrt{2 L_{x} L_{y}}}\left(\begin{array}{l}
1 \\
i
\end{array}\right) e^{i(\mathbf{Q}+\mathbf{q}) \cdot \mathbf{r}}\left(\frac{1}{\pi l_{z}^{2}}\right)^{1 / 4} e^{-\xi_{q}^{2} / 2}
$$

Here, $\xi_{q}=\left(z+z_{q}\right) / l_{z}, z_{q}=q l_{B}^{2}$, and the pseudomagnetic localization length in the $z$ direction is given by

$$
l_{z}=\sqrt{\frac{t_{2}}{t Q}} l_{B}
$$

Alternatively, one can perform a gauge transformation such that the wave function is localized in the $x-y$ plane and a plane wave in the $z$ direction. In that case, the localization length is

$$
l_{x y}=\frac{l_{B}^{2}}{l_{z}} .
$$

The prefactors in the localization lengths arise due to the elongations of the elliptical cyclotron orbits.

Going beyond the lowest-order expansion in $Q$ would lead to a $\theta$ dependence of the strength of the pseudomagnetic field because the strain shrinks the nodal line 
anisotropically. Nevertheless, the energy of the zeroth PLL is independent of the strength of the pseudomagnetic field, and hence, in all cases, we robustly obtain the desired 3D flat band. Since the pseudomagnetic field originates from the spatial variation of the radius of the nodal loop, it can also be created by varying the relative amount of $\mathrm{P}$ and As contents in the $\mathrm{CaAgP}_{1-x} \mathrm{As}_{x}$ alloys along the $z$ direction, for example, by changing the crystal growth conditions with time.

\section{Connection of drumhead surface states and pseudo-Landau level}

In Figs. 1(c) and 1(d), we plot the spectrum of the lowest PLL states. We find that the zeroth PLL is twofold degenerate: The bulk states discussed above coexist with drumhead states localized to the bottom surface. We further observe that the PLL bulk states evolve from drumhead states localized to the top surface, similar to the connection between Fermi arcs and PLLs in Weyl semimetals [40]. By using approximate analytic solutions for the drumhead surface states and for the PLL bulk wave functions, and by considering their coupling at the surface of the sample, we obtain an effective low-energy Hamiltonian (see Supplemental Material [38]) that accurately describes the exact numerical results as shown in Figs. 1(d) and 1(e). We confirm the 3D nature of the flat band by plotting the scaling of the total number of states in the flat band for different thicknesses $L$ with and without strain in Fig. 1(f). For sufficiently large $L$, the number of states for the drumhead surface states without strain saturates to the value $N_{0}=Q^{2} L_{x} L_{y} /(2 \pi)$. On the contrary, the number of states, including the PLL bulk states, in the presence of strain grows linearly with $L$ as $N_{\text {flat }}=Q L_{x} L_{y} L /\left(2 \pi l_{B}^{2}\right)$ confirming our hypothesis of a flat band that is genuinely $3 \mathrm{D}$.

The interaction effects depend on the k-space extent of the flat bands. Unlike in twisted bilayer graphene, the flat band in these 3D systems covers only a fraction of the Brillouin zone. In twisted bilayer graphene, however, the area of the moiré-Brillouin zone is inversely proportional to the square of the superlattice lattice constant, and the latter is large around the magic angle. Thus, we expect that interaction effects in the considered 3D flat-band systems are as important as in the case of twisted bilayer graphene (see below).

\section{EFFECTS OF INTERACTIONS}

In flat-band systems, the density of states diverges, and therefore, interaction effects are important. Moreover, these systems have instabilities with respect to both repulsive and attractive interactions, so competition between various symmetry-broken phases is expected to be a generic feature of flat-band systems. We now turn our attention to the effect of an attractive pairing interaction and Coulomb repulsion between electrons.

\section{A. Magnetism}

For the study of magnetism, we self-consistently solve the mean-field equations for the magnetic-order parameter $m_{z}$ and the chemical potential $\mu$ in the presence of the density constraint that the filling factor of the band be fixed to $C$ (see Supplemental Material [38]),

$$
\begin{aligned}
m_{z} & =\frac{V_{0}}{2}\left[n_{F}\left(-m_{z}-\mu\right)-n_{F}\left(m_{z}-\mu\right)\right], \\
C & =n_{F}\left(-m_{z}-\mu\right)+n_{F}\left(m_{z}-\mu\right) .
\end{aligned}
$$

Here, $V_{0}$ is the effective interaction strength, and $n_{F}$ is the Fermi function. The filling factor $C$ is restricted to $0 \leq C \leq 2$, such that $C=0$ corresponds to the situation where the flat-band states are completely empty, whereas for $C=2$, both spin-up and spin-down flat-band states are fully occupied. The effective interaction strength $V_{0}$ is computed by projecting the Coulomb interaction to the zeroth PLL. Furthermore, it can be tuned by varying the strain (see Supplemental Material [38]). The critical temperature for magnetism depends on $C$ as

$$
k_{B} T_{c, m}=\frac{V_{0}}{4} C(2-C) .
$$

For conservative model parameters, we estimate $T_{c, m}=3 \mathrm{~K}$ (see Supplemental Material [38]).

We point out that this theory describes several different types of magnetic-order parameters, as their projections to the PLL bulk wave functions are the same (see Supplemental Material [38]). However, the surface effects break the symmetry explicitly and distinguish the magnetic-order parameters from each other. We find that the lowest energy state corresponds to the situation where the magnetic order is staggered with respect to the PLL bulk states and the drumhead states at the bottom surface (see Supplemental Material [38]). We note that the exact nature of the magnetic order is not important for the discussion of competing phases below since we focus on the bulk states here.

\section{B. Superconductivity}

Starting from the reduced BCS Hamiltonian with the density constraint, the mean-field equations for the superconducting order parameter $\Delta$ and the chemical potential $\mu$ are (see Supplemental Material [38])

$$
\begin{aligned}
& \Delta=G_{0} \frac{\Delta}{2 \sqrt{\mu^{2}+\Delta^{2}}} \tanh \left(\beta \sqrt{\Delta^{2}+\mu^{2}} / 2\right), \\
& C=1+\frac{\mu}{\sqrt{\mu^{2}+\Delta^{2}}} \tanh \left(\beta \sqrt{\Delta^{2}+\mu^{2}} / 2\right),
\end{aligned}
$$


where $G_{0}$ is the effective interaction strength and $\beta=1 /\left(k_{B} T\right)$. The critical temperature is given by

$$
k_{B} T_{c, s c}=\frac{G_{0}}{4} \frac{C-1}{\operatorname{arctanh}(C-1)} .
$$

For typical model parameters, we estimate $T_{c, s c}=1 \mathrm{~K}$ (see Supplemental Material [38]).

We note that this value is similar to the critical temperatures observed in twisted bilayer graphene [10,11]. In the case of 3D flat bands in NLSMs, however, the stronger suppression of order-parameter fluctuations and the greater variability of parameters with strain can potentially lead to even larger critical temperatures. The same applies to the magnetic phase described above.

\section{Competing phases}

As recent experiments in twisted bilayer graphene indicate, the competition between different correlated phases is a generic feature of flat-band systems $[15,16]$. Moreover, in the case of competition between flat-band magnetism and superconductivity doping, the system away from half-filling is generically expected to favor the superconducting phase [8]. Thus, we also expect magnetic and superconducting phases in 3D flat-band systems to be tunable using controllable parameters such as doping and the effective interaction strengths. The latter can be controlled, for example, by strain or an electrostatic environment.

This expectation is confirmed by our calculations shown in Fig. 2. In Fig. 2(a), we plot the critical temperatures for magnetism [Eq. (4)] and superconductivity [Eq. (6)] as a function of the filling factor $C$. At half-filling $C=1$, the system realizes the phase with the larger effective interaction strength. Hence, for the typical situation where the Coulomb repulsion dominates the pairing interaction, $V_{0}>G_{0}$, the magnetic phase is realized. On the contrary, doping the system away from half-filling favors the superconducting phase. Therefore, flat-band systems are expected to have phase diagrams similar to those observed experimentally in cuprates [41] and twisted bilayer graphene $[10,11]$. In contrast to the complicated, strongly correlated Mott physics of cuprates [41], here the mechanisms of superconductivity and magnetism are independent of each other [9], which is in agreement with experiments on twisted bilayer graphene $[15,16]$. In addition to the filling factor $C$, the ground state can also be controlled by the relative interaction strength $G_{0} / V_{0}$, as shown in Fig. 2(b).

We note that our proposal offers a degree of control over $G_{0}$ and $V_{0}$ in a single sample. By varying the strength of the strain, one changes the parameters as derived in the Supplemental Material [38], thus allowing us to tune in situ between magnetic and superconducting phases.
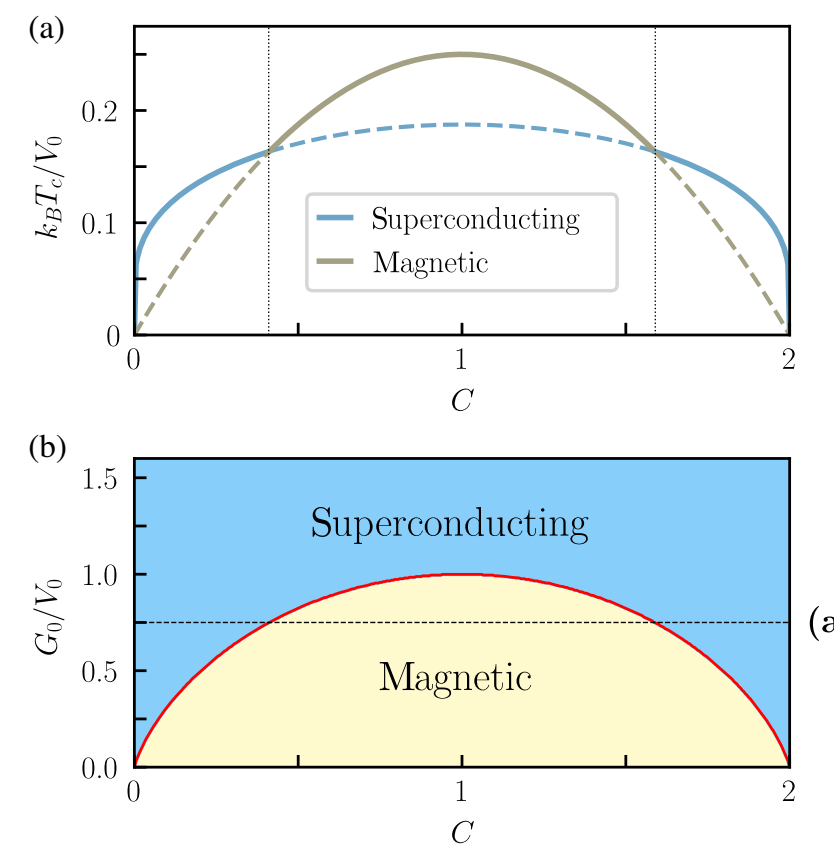

(a)

FIG. 2. Phase diagram of the model Hamiltonian [Eq. (1)] in the presence of strain [Eq. (2)] and interactions [Eqs. (3) and (5)]. (a) Critical temperatures for magnetism [Eq. (4)] and superconductivity [Eq. (6)] as a function of the filling factor $C$ in the typical situation where the effective interaction strength for superconductivity is smaller than for magnetism $G_{0}=0.75 V_{0}$. At half-filling $C=1$, the system realizes magnetic order, but doping away from half-filling leads to superconductivity, resembling the phase diagrams experimentally observed in cuprates and twisted bilayer graphene. (b) Phase diagram as a function of $C$ and $G_{0} / V_{0}$, assuming that the system realizes the phase with the larger critical temperature. The dashed horizontal line represents a path through the phase diagram corresponding to panel (a).

We note that, in reality, the phase diagram may be more complicated because of the possible coexistence of the order parameters near the mean-field phase boundary [9], which may be analyzed using numerical techniques or the functional renormalization group and are beyond the scope of the current paper. Nevertheless, we underline that 3D flat bands can shed light on the competition and intertwining of order parameters while avoiding the complications caused by strongly correlated Mott physics and the fluctuations present in low-dimensional systems.

\section{Superfluid stiffness}

To further substantiate our claims concerning the stability of the mean-field solutions, we analyze the collective modes of the system. In the case of 3D flat bands, the quasiparticle spectrum is fully gapped, which means that the amplitude mode is gapped. Thus, the order parameter is stable against amplitude-mode fluctuations, which is in strong contrast to the order parameter appearing in the case of flat-band superconductivity due to 2D drumhead surface 
states, where the system is gapless and susceptible to strong amplitude-mode fluctuations [42].

The phase rigidity, on the other hand, needs to be analyzed more carefully as the kinetic contribution to it is negligible because of the flatness of the band. It is determined by the superfluid stiffness tensor $D_{s}$, which is related to the supercurrent $\mathbf{j}$ in the system as

$$
j_{i}=\frac{2 e}{\hbar} \sum_{j}\left[D_{s}\right]_{i j}\left(\partial_{j} \varphi-\frac{2 e}{\hbar} A_{j}\right),
$$

where $\varphi$ is the phase of the superconducting order parameter. The question of whether a supercurrent even exists in the systems considered here is particularly relevant as the Fermi velocity within a featureless flat band is zero. Previous studies have shown that in 2D systems, there can still exist a nonzero contribution to the superfluid stiffness caused by the quantum geometry of the Bloch wave functions [6,43-49]. However, the possibility of an analogous $3 \mathrm{D}$ quantum geometry that gives rise to superfluid stiffness in all directions of a 3D system has not been explored. In the Supplemental Material [38], we combine methods developed in the context of quantum Hall systems $[6,43]$ and flat-band superconductors $[44,47]$ to calculate the superfluid stiffness for the model Hamiltonian [Eq. (1)] in the presence of strain [Eq. (2)]. We find that the superfluid stiffness perpendicular, $D_{s, z z}$, and parallel, $D_{s, x x}=D_{s, y y}$, to the layers have large geometric contributions

$$
D_{s, z z}=\frac{n_{0} l_{z}^{2}}{2} \Delta, \quad D_{s, x x}=D_{s, y y}=\Delta \frac{n_{0}}{4} l_{x y}^{2} .
$$

Therefore, the system has rigidity against phase fluctuations and supports large bulk supercurrents, thereby confirming the 3D nature of the superconducting state. Here, $n_{0}=Q /\left(2 \pi l_{B}^{2}\right)$ is the degeneracy of the flat band per volume, and the directional dependence of the stiffness arises because of the elongations of the semiclassical cyclotron orbits.

We emphasize that the nonzero superfluid stiffness along all three directions, as obtained in this section, is not a property that flat bands exhibit by default, but the necessary 3D quantum geometry arises because of the variation of the pseudomagnetic field direction along the nodal line [Eq. (2) and Supplemental Material [38] ].

\section{MATERIAL CONSIDERATIONS}

In this section, we provide a materials perspective on our proposal. In particular, we identify $\mathrm{CaAgP}$ and rhombohedral graphite NLSMs as possible material platforms for 3D flat bands with a negligible spin-orbit interaction. We note that NLSMs can also be realized in systems with sizable spin-orbit interactions. Theoretically proposed candidates are $\mathrm{TlTaSe}_{2}$ [50] or alloys of SnTe [51].

\section{A. Strained CaAgP}

$\mathrm{CaAgP}$ crystallizes in a ZrNiAl-type hexagonal structure [52], which is illustrated in Fig. 3(a). Standard DFT calculations predict a topological NLSM phase for the nodal-line Dirac semimetal CaAgP. However, standard DFT is known to overestimate the band inversion and, in fact, experimental results for $\mathrm{CaAgP}$ show a trivially gapped phase for this compound. Nonetheless, it has been shown both theoretically and experimentally that strain and As doping can turn the phase from trivial to topological [53]. In the following, we focus on the NLSM phase of the $\mathrm{CaAgP}$ materials class.

The low-energy band structure is formed by the $5 s$ orbitals of the $\mathrm{Ag}$ atoms and the $3 p$ orbitals of the light $\mathrm{P}$ atoms. Therefore, spin-orbit interaction in $\mathrm{CaAgP}$ is small. In the atomic limit, the $p$ orbitals are occupied, and the $s$ orbitals are unoccupied. At the $\Gamma$ and at the A point of the Brillouin zone, there is a band inversion between one $s$ band and one $p_{z}$ band, while the band orderings at $\mathrm{M}$ and $\mathrm{K}$ are trivial. As a consequence, a line node is observed along the paths $\Gamma-\mathrm{M}$ and $\Gamma-\mathrm{K}$, which forms a circle in the $k_{x}-k_{y}$ plane centered at the $\Gamma$ point [see Fig. 3(a) and Supplemental Material [38] ]. The band dispersion at the line node is linear along both the radial and the $k_{z}$ directions. It should be noted that this line node is generally not protected from spin-orbit interactions, but in this case, the weak spin-orbit coupling induces only a small gap on the order of $10 \mathrm{~K}$ [52].

We construct an effective two-orbital tight-binding model (see Supplemental Material [38]) based on one $3 p_{z}$-orbital Wannier function (WF) centered at one $\mathrm{P}$ atom, and one $5 s$-orbital WF centered within a triangle of $\mathrm{Ag}$ atoms as previously done for other triangular systems [54]. With an energy cutoff of $6 \mathrm{meV}$ for the tight-binding parameters, this allows us to accurately capture the lowenergy physics with a relatively simple model. Most importantly, our two-band model correctly reproduces the NLSM phase of the CaAgP materials class [see Fig. 3(b)].

In the next step, we implement the strain terms by modifying the tight-binding parameters in a way similar to our minimal model above (see Supplemental Material [38]). We choose the bending direction to be aligned with the $x$ axis. We compute the energy spectrum for a (001) slab and find two nearly flat bands, as shown in Fig. 3(c). Similar to our minimal model, these bands consist of superpositions of bottom-surface states and PLL bulk states. In particular, the center of the PLL bulk states shifts as a function of the flat-band momentum, which happens symmetrically around the center of the flat band at $\bar{\Gamma}$. However, because of the broken electron-hole symmetry in this material, the two flat bands acquire a small dispersion. 
(a)

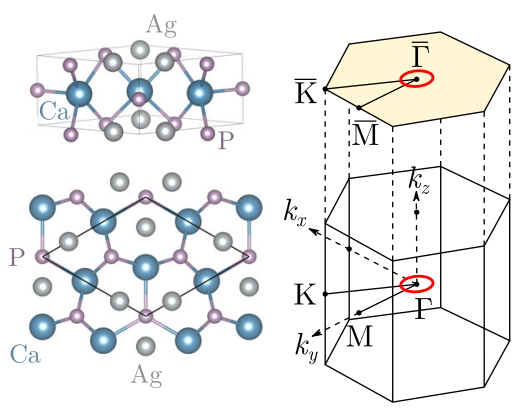

(d)

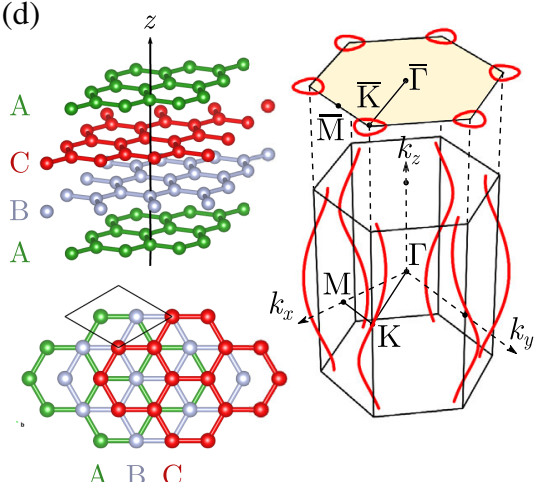

(b)

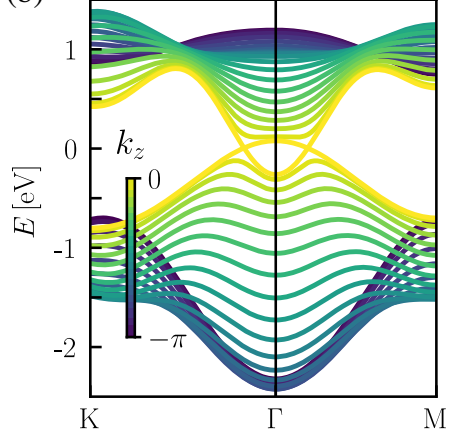

(e)

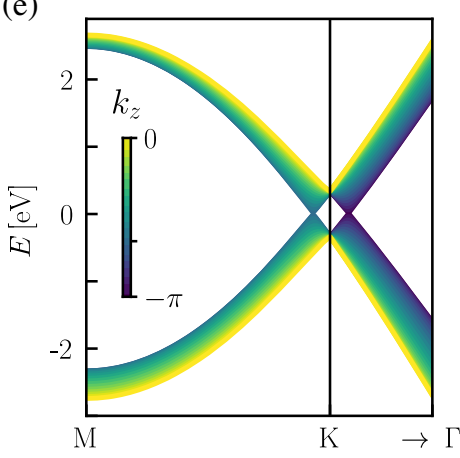

(c)

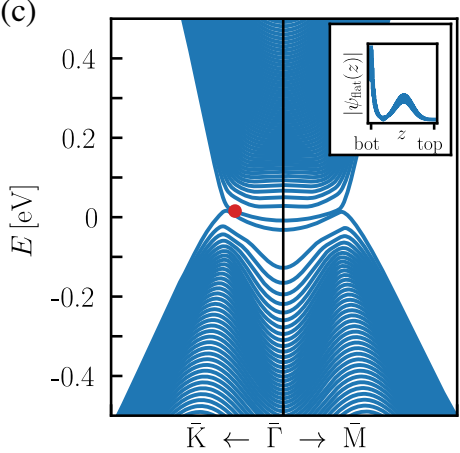

(f)

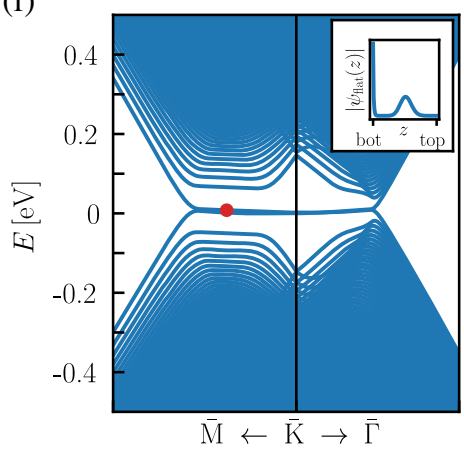

FIG. 3. Three-dimensional flat bands in nodal-line semimetals CaAgP (first row) and rhombohedral graphite (second row). (a) Top and side views of the CaAgP crystal structure. Next to these, we indicate the shape and position of its nodal circle (red), which is centered at the $\Gamma$ point of the hexagonal Brillouin zone. The beige hexagon represents the (001) surface Brillouin zone with the surface projection of the nodal loop. (b) Bulk bands of the two-band tight-binding model without strain along paths with fixed $k_{z}$. The bands only cross in the plane $k_{z}=0$. (c) Tight-binding spectrum of a strained (001) slab for $L=200, R=800$ with nearly flat bands. The inset shows the spatial profile of the flat-band wave function at the momentum indicated by the red dot. It is a superposition of a bottom-surface state and a (Gaussian) Landau-level state. (d) Top and side views of ABC-stacked (rhombohedral) graphite next to a sketch of its nodal lines. In contrast to $\mathrm{CaAgP}$, there are several nodal lines that spiral around axes going through the $\mathrm{K}$ and $\mathrm{K}$ ' points of the hexagonal Brillouin zone. Their projections into the surface Brillouin zone form approximate nodal circles. (e) Bulk bands of the two-band tight-binding model without strain along paths with fixed $k_{z}$. The nodal spiral crosses the considered path for two different values of $k_{z}$. (f) Tightbinding spectrum of a strained (001) slab for $L=100, R=400$ with flat bands. The inset shows the spatial profile of the flat-band state at the red dot, which is again composed of a Landau-level and a bottom-surface state.

Furthermore, we observe a small energy splitting between the two bands due to chiral-symmetry breaking, a symmetry that was present in the minimal model above because of its simplicity. The corresponding gap is smaller in the graphite case that we turn to now.

\section{B. Strained rhombohedral graphite}

Another possible candidate material is rhombohedral graphite. It consists of light carbon atoms and therefore has negligible spin-orbit interaction. Rhombohedral graphite is composed of ABC-stacked graphene sheets [see Fig. 3(d)]. Isolated graphene sheets feature Dirac cones at the $\mathrm{K}$ and $\mathrm{K}$ ' points of their 2D Brillouin zone. Stacking several sheets in the $z$ direction in an ABC-type fashion leads to a $k_{z}$-dependent shift of the Dirac cones away from the $\mathrm{K}$ and $\mathrm{K}$ ' points, thereby forming a nodal line. In particular, the nodal lines in rhombohedral graphite spiral around the vertical hinges of its hexagonal Brillouin zone [see Fig. 3(d)].

The low-energy properties of rhombohedral graphite, similar to graphene, can be captured by a two-band model (see Supplemental Material [38]). This model reproduces the nodal spirals as can be inferred from comparing the spectrum shown in Fig. 3(e) to the Brillouin zone sketch in Fig. 3(d). Note that the electron-hole symmetry is only weakly broken in this material.

As before, we implement strain terms corresponding to a cylindrical strain profile with the bending direction aligned with the $x$ axis (see Supplemental Material [38]). The energy spectrum of a (001) slab of this system features two flat bands at the Fermi level, as illustrated in Fig. 3(f). In contrast to $\mathrm{CaAgP}$, their splitting and bandwidth is negligibly small because of the approximate electron-hole symmetry of the material. The flat bands are again composed of PLL bulk states and drumhead surface states. 
However, their behavior and composition as a function of the flat-band momentum $\left(q_{x}, q_{y}\right)$ differs from our previous observations. Depending on the direction, we observe all possible combinations: bottom-surface and PLL bulk state, PLL bulk and top-surface state, bottom- and top-surface state, and even two PLL bulk states with opposite shift behavior (see Supplemental Material [38]). Despite these differences, we emphasize that, in all cases, a 3D flat band appears robustly in the presence of strain.

\section{DISCUSSION AND CONCLUSIONS}

We have proposed a feasible way to create 3D flat bands by applying strain to a nodal-line semimetal. In the process, we have discovered an inherent connection between the arising 3D flat band and the drumhead surface states of the parent nodal-line semimetal. Moreover, we have investigated the effects of interactions on the flat bands, highlighting the competition of superconductivity and magnetism in analogy with twisted bilayer graphene. By computing the superfluid stiffness, we have confirmed that the flatness of the bands does not impede the phase rigidity of the system. The flat-band system thus supports supercurrents and true long-range order solely due to the 3D quantum geometry of the Bloch wave functions, which arises because of the variation of the pseudomagnetic field direction along the nodal line. Going beyond our general idea, we have applied our theory to the potential candidate materials $\mathrm{CaAgP}$ and rhombohedral graphite. We have shown that both materials give rise to sufficiently flat bands under experimentally accessible strain, therefore representing viable candidate materials for the experimental realization of our ideas. Our conservative estimates for the critical temperatures are on the order of a few Kelvins. However, we emphasize that they depend strongly on the magnitude of the applied strain or the pseudomagnetic field. In particular, if the pseudomagnetic field is controlled with the chemical composition, we expect that much stronger pseudofield strengths can be obtained, such that critical temperatures on the order of tens of Kelvins could potentially be achieved. The critical temperatures are proportional to the $\mathbf{k}$-space extent of the flat bands. Therefore, it is important to note that, even though the portion of the Brillouin zone covered by the flat band is small under the assumptions $Q \ll 1$ and $R \gg L$, the flat bands can generally cover an arbitrary portion of the Brillouin zone. In particular, the first assumption $Q \ll 1$ was made only for the sake of analytical transparency. Moreover, implementing the effective magnetic field by varying the chemical composition so that the radius of the nodal line changes along the $z$ direction, we may also violate the second condition $R \gg L$. In the extreme case $Q \sim 1$ and $R \sim L$, we find numerically that the 3D flat bands can indeed cover most of the Brillouin zone (see Supplemental Material in Ref. [38]).
We have considered a specific geometry, realizable by bending the sample, leading to a suitable strain profile. However, we have explicitly checked that only the strain tensor component $u_{11}$ matters for the appearance of the 3D flat band (see Supplemental Material in Ref. [38]), and therefore, the only important ingredient for realizing a 3D flat band in nodal-line semimetals is an inhomogeneous tensile strain profile across the sample. Thus, a suitable strain tensor can also be realized in various other ways, such as by utilizing the type of multilayer structure designed in Ref. [55] for the creation of a giant linear strain gradient.

We emphasize that if the strain is realized by bending the sample, the different phases and phase transitions can be studied in situ in this system. As discussed above, tuning the strain changes the relative interaction strengths of superconductivity and magnetism, thereby allowing us to study the transition between these phases. Moreover, it is known that flat bands are an interesting playground for Anderson localization [56]. The in situ tunability of the flatband degeneracy by strain also controls the effective disorder strength, so it might be possible to study the Anderson transition in a single sample.

In condensed-matter physics, there is immediate interest for the experimental community to realize this $3 \mathrm{D}$ analogue of twisted bilayer graphene in order to study the interplay of quantum geometry, flatness of the dispersion, disorder, and intertwining of different types of order. Moreover, our proposal of realizing 3D flat bands by strain engineering nodal-line semimetals opens a new frontier of research beyond condensed-matter physics because metamaterials and cold atomic gas systems can be engineered to exhibit dispersion relations of topological semimetals to a large precision [57,58]. Controllable interactions in the cold atom systems make realization of superconductivity and magnetism per our predictions possible in such systems. Beyond that, there are many related conceptual questions: How general is the connection between the surface states and the lowest pseudo-Landau levels in the presence of a gauge field. Can the picture be expanded to other semimetals and probably even to topological insulators, superconductors, and weak and fragile phases? We also note that Dirac nodal lines in the presence of effective gauge fields can lead to peculiar effective electrodynamics [59], and it might be interesting to study the connection to surface states also in this context.

The data shown in the figures and the code generating all of the data are available in Ref. [60].

\section{ACKNOWLEDGMENTS}

We thank Raquel Queiroz, Leslie Schoop, Roni Ilan, and Adolfo Grushin for useful discussions. This work was supported by the Foundation for Polish Science through the International Research Agendas program co-financed by 
the European Union within the Smart Growth Operational Programme. We acknowledge access to the computing facilities of the Interdisciplinary Center of Modeling at the University of Warsaw, Grants No. G73-23, No. G75-10, and No. G84-4.

[1] S. T. Belyaev, On the Nature of the First Excited States of Even-Even Spherical Nuclei, ZhETF 39, 1387 (1961) [J. Exp. Theor. Phys. 12, 968 (1961)], http:// www.jetp.ac .ru/cgi-bin/e/index/e/12/5/p968?a=list.

[2] R. W. Richardson and N. Sherman, Exact Eigenstates of the Pairing-Force Hamiltonian, Nucl. Phys. 52, 221 (1964).

[3] J. Dukelsky, S. Pittel, and G. Sierra, Colloquium: Exactly Solvable Richardson-Gaudin Models for Many-Body Quantum Systems, Rev. Mod. Phys. 76, 643 (2004).

[4] J. von Delft and D. C. Ralph, Spectroscopy of Discrete Energy Levels in Ultrasmall Metallic Grains, Phys. Rep. 345, 61 (2001).

[5] J. K. Jain, Composite Fermions (Cambridge University Press, Cambridge, England, 2007).

[6] K. Moon, H. Mori, Kun Yang, S. M. Girvin, A. H. MacDonald, L. Zheng, D. Yoshioka, and S.-C. Zhang, Spontaneous Interlayer Coherence in Double-Layer Quantum Hall Systems: Charged Vortices and KosterlitzThouless Phase Transitions, Phys. Rev. B 51, 5138 (1995).

[7] N. B. Kopnin, T. T. Heikkilä, and G. E. Volovik, HighTemperature Surface Superconductivity in Topological Flat-Band Systems, Phys. Rev. B 83, 220503(R) (2011).

[8] T. Löthman and A. M. Black-Schaffer, Universal Phase Diagrams with Superconducting Domes for Electronic Flat Bands, Phys. Rev. B 96, 064505 (2017).

[9] R. Ojajärvi, T. Hyart, M. A. Silaev, and T. T. Heikkilä, Competition of Electron-Phonon Mediated Superconductivity and Stoner Magnetism on a Flat Band, Phys. Rev. B 98, 054515 (2018).

[10] Y. Cao, V. Fatemi, S. Fang, K. Watanabe, T. Taniguchi, E. Kaxiras, and P. Jarillo-Herrero, Unconventional Superconductivity in Magic-Angle Graphene Superlattices, Nature (London) 556, 43 (2018).

[11] Y. Cao, V. Fatemi, A. Demir, S. Fang, S. L. Tomarken, J. Y. Luo, J. D. Sanchez-Yamagishi, K. Watanabe, T. Taniguchi, E. Kaxiras, R. C. Ashoori, and P. Jarillo-Herrero, Correlated Insulator Behaviour at Half-Filling in Magic-Angle Graphene Superlattices, Nature (London) 556, 80 (2018).

[12] X. Lu, P. Stepanov, W. Yang, M. Xie, M. A. Aamir, I. Das, C. Urgell, K. Watanabe, T. Taniguchi, G. Zhang, A. Bachtold, A. H. MacDonald, and D. K. Efetov, Superconductors, Orbital Magnets and Correlated States in Magic-Angle Bilayer Graphene, Nature (London) 574, 653 (2019).

[13] M. Serlin, C. L. Tschirhart, H. Polshyn, Y. Zhang, J. Zhu, K. Watanabe, T. Taniguchi, L. Balents, and A. F. Young, Intrinsic Quantized Anomalous Hall Effect in a Moiré Heterostructure, Science 367, 900 (2020).

[14] G. Chen, A. L. Sharpe, E. J. Fox, Y.-H. Zhang, S. Wang, L. Jiang, B. Lyu, H. Li, K. Watanabe, T. Taniguchi, Z. Shi, T. Senthil, D. Goldhaber-Gordon, Y. Zhang, and F. Wang, Tunable Correlated Chern Insulator and Ferromagnetism in a Moiré Superlattice, Nature (London) 579, 56 (2020).
[15] P. Stepanov, I. Das, X. Lu, A. Fahimniya, K. Watanabe, T. Taniguchi, F. H. L. Koppens, J. Lischner, L. Levitov, and D. K. Efetov, Untying the Insulating and Superconducting Orders in Magic-Angle Graphene, Nature (London) 583, 375 (2020).

[16] Y. Saito, J. Ge, K. Watanabe, T. Taniguchi, and A. F. Young, Independent Superconductors and Correlated Insulators in Twisted Bilayer Graphene, Nat. Phys. 16, 926 (2020).

[17] C. H. Lee, D. P. Arovas, and R. Thomale, Band Flatness Optimization through Complex Analysis, Phys. Rev. B 93, 155155 (2016).

[18] J.-X. Yin, S. S. Zhang, G. Chang, Q. Wang, S. S. Tsirkin, Z. Guguchia, B. Lian, H. Zhou, K. Jiang, I. Belopolski, N. Shumiya, D. Multer, M. Litskevich, T. A. Cochran, H. Lin, Z. Wang, T. Neupert, S. Jia, H. Lei, and M.Z. Hasan, Negative Flat Band Magnetism in a Spin-Orbit-Coupled Correlated Kagome Magnet, Nat. Phys. 15, 443 (2019).

[19] D.-S. Ma, Y. Xu, C. S. Chiu, N. Regnault, A. A. Houck, Z. Song, and B. A. Bernevig, Spin-Orbit-Induced Topological Flat Bands in Line and Split Graphs of Bipartite Lattices, Phys. Rev. Lett. 125, 266403 (2020).

[20] V. A. Khodel' and V. R. Shaginyan, Superfluidity in System with Fermion Condensate, JETP Lett. 51, 553 (1990), http:// jetpletters.ru/ps/1143/article_17312.shtml.

[21] Y. Fu, E. J. König, J.H. Wilson, Y.-Z. Chou, and J. H. Pixley, Magic-Angle Semimetals, npj Quantum Mater. 5, 71 (2020).

[22] C.-K. Chiu, J. C. Y. Teo, A. P. Schnyder, and S. Ryu, Classification of Topological Quantum Matter with Symmetries, Rev. Mod. Phys. 88, 035005 (2016).

[23] R. Ilan, A. G. Grushin, and D. I. Pikulin, PseudoElectromagnetic Fields in 3D Topological Semimetals, Nat. Rev. Phys. 2, 29 (2020).

[24] N. Levy, S. A. Burke, K. L. Meaker, M. Panlasigui, A. Zettl, F. Guinea, A. H. C. Neto, and M. F. Crommie, StrainInduced Pseudo-Magnetic Fields Greater than 300 Tesla in Graphene Nanobubbles, Science 329, 544 (2010).

[25] E. Tang and L. Fu, Strain-Induced Partially Flat Band, Helical Snake States and Interface Superconductivity in Topological Crystalline Insulators, Nat. Phys. 10, 964 (2014).

[26] B. Amorim, A. Cortijo, F. de Juan, A. G. Grushin, F. Guinea, A. Gutiérrez-Rubio, H. Ochoa, V. Parente, R. Roldán, P. San-Jose, J. Schiefele, M. Sturla, and M. A. H. Vozmediano, Novel Effects of Strains in Graphene and Other Two Dimensional Materials, Phys. Rep. 617, 1 (2016).

[27] V. J. Kauppila, F. Aikebaier, and T. T. Heikkilä, Flat-Band Superconductivity in Strained Dirac Materials, Phys. Rev. B 93, 214505 (2016).

[28] V. Peri, M. Serra-Garcia, R. Ilan, and S. D. Huber, AxialField-Induced Chiral Channels in an Acoustic Weyl System, Nat. Phys. 15, 357 (2019).

[29] S. W. Kim and B. Uchoa, Elastic Gauge Fields and ZeroField Three-Dimensional Quantum Hall Effect in Hyperhoneycomb Lattices, Phys. Rev. B 99, 201301(R) (2019).

[30] A. L. R. Manesco, J. L. Lado, E. V. Ribeiro, G. Weber, and D. Rodrigues, Jr., Correlations in the Elastic Landau Level of Spontaneously Buckled Graphene, 2D Mater. 8, 015011 (2021). 
[31] T. D. C. Bevan, A. J. Manninen, J. B. Cook, J. R. Hook, H. E. Hall, T. Vachaspati, and G. E. Volovik, Momentum Creation by Vortices in Superfluid ${ }^{3} \mathrm{He}$ as a Model of Primordial Baryogenesis, Nature (London) 386, 689 (1997).

[32] C.-X. Liu, P. Ye, and X.-L. Qi, Chiral gauge field and axial anomaly in a Weyl semimetal, Phys. Rev. B 87, 235306 (2013).

[33] H. Shapourian, T. L. Hughes, and S. Ryu, Viscoelastic Response of Topological Tight-Binding Models in Two and Three Dimensions, Phys. Rev. B 92, 165131 (2015).

[34] E. Fradkin, S. A. Kivelson, and J. M. Tranquada, Colloquium: Theory of Intertwined Orders in High Temperature Superconductors, Rev. Mod. Phys. 87, 457 (2015).

[35] L. S. Xie, L. M. Schoop, E. M. Seibel, Q. D. Gibson, W. Xie, and R. J. Cava, A New Form of $\mathrm{Ca}_{3} \mathrm{P}_{2}$ with a Ring of Dirac Nodes, APL Mater. 3, 083602 (2015).

[36] Y.-H. Chan, C.-K. Chiu, M. Y. Chou, and A. P. Schnyder, $\mathrm{Ca}_{3} \mathrm{P}_{2}$ and Other Topological Semimetals with Line Nodes and Drumhead Surface States, Phys. Rev. B 93, 205132 (2016).

[37] Y. Wang and R. M. Nandkishore, Topological Surface Superconductivity in Doped Weyl Loop Materials, Phys. Rev. B 95, 060506(R) (2017).

[38] See Supplemental Material at http://link.aps.org/ supplemental/10.1103/PhysRevX.11.031017 for details of the pseudomagnetic field, analytical expressions of bulkand surface-state wave functions of the zeroth pseudoLandau level, the mean-field theory of the arising magnetic and superconducting phases, the in-plane and out-of-plane superfluid stiffness, the tight-binding model and wavefunction expressions of rhombohedral graphite, and details on the DFT calculations of $\mathrm{CaAgP}$, as well as for how the strain was implemented in the low-energy models.

[39] T. Liu, D. I. Pikulin, and M. Franz, Quantum Oscillations without Magnetic Field, Phys. Rev. B 95, 041201(R) (2017).

[40] A. G. Grushin, J. W. F. Venderbos, A. Vishwanath, and R. Ilan, Inhomogeneous Weyl and Dirac Semimetals: Transport in Axial Magnetic Fields and Fermi Arc Surface States from Pseudo-Landau Levels, Phys. Rev. X 6, 041046 (2016).

[41] P. A. Lee, N. Nagaosa, and X.-G. Wen, Doping a Mott Insulator: Physics of High-Temperature Superconductivity, Rev. Mod. Phys. 78, 17 (2006).

[42] V. J. Kauppila, T. Hyart, and T. T. Heikkilä, Collective Amplitude Mode Fluctuations in a Flat Band Superconductor Formed at a Semimetal Surface, Phys. Rev. B 93, 024505 (2016).

[43] D. I. Pikulin, P. G. Silvestrov, and T. Hyart, ConfinementDeconfinement Transition Due to Spontaneous Symmetry Breaking in Quantum Hall Bilayers, Nat. Commun. 7, 10462 (2016).

[44] S. Peotta and P. Törmä, Superfluidity in Topologically Nontrivial Flat Bands, Nat. Commun. 6, 8944 (2015).

[45] L. Liang, T. I. Vanhala, S. Peotta, T. Siro, A. Harju, and P. Törmä, Band Geometry, Berry Curvature, and Superfluid Weight, Phys. Rev. B 95, 024515 (2017).

[46] X. Hu, T. Hyart, D. I. Pikulin, and E. Rossi, Geometric and Conventional Contribution to the Superfluid Weight in
Twisted Bilayer Graphene, Phys. Rev. Lett. 123, 237002 (2019).

[47] F. Xie, Z. Song, B. Lian, and B. A. Bernevig, TopologyBounded Superfluid Weight in Twisted Bilayer Graphene, Phys. Rev. Lett. 124, 167002 (2020).

[48] A. Julku, T. J. Peltonen, L. Liang, T. T. Heikkilä, and P. Törmä, Superfluid Weight and Berezinskii-KosterlitzThouless Transition Temperature of Twisted Bilayer Graphene, Phys. Rev. B 101, 060505(R) (2020).

[49] X. Hu, T. Hyart, D. I. Pikulin, and E. Rossi, QuantumMetric-Enabled Exciton Condensate in Double Twisted Bilayer Graphene, arXiv:2008.03241.

[50] G. Bian, T.-R. Chang, H. Zheng, S. Velury, S.-Y. Xu, T. Neupert, C.-K. Chiu, S.-M. Huang, D. S. Sanchez, I. Belopolski, N. Alidoust, P.-J. Chen, G. Chang, A. Bansil, H.-T. Jeng, H. Lin, and M. Z. Hasan, Drumhead Surface States and Topological Nodal-Line Fermions in $\mathrm{TlTaSe}_{2}$, Phys. Rev. B 93, 121113(R) (2016).

[51] A. Lau and C. Ortix, Topological Semimetals in the SnTe Material Class: Nodal Lines and Weyl Points, Phys. Rev. Lett. 122, 186801 (2019).

[52] A. Yamakage, Y. Yamakawa, Y. Tanaka, and Y. Okamoto, Line-Node Dirac Semimetal and Topological Insulating Phase in Noncentrosymmetric Pnictides $\operatorname{CaAg} X(X=P$, As), J. Phys. Soc. Jpn. 85, 013708 (2016).

[53] N. Xu, Y. T. Qian, Q. S. Wu, G. Autès, C. E. Matt, B. Q. Lv, M. Y. Yao, V. N. Strocov, E. Pomjakushina, K. Conder, N. C. Plumb, M. Radovic, O. V. Yazyev, T. Qian, H. Ding, J. Mesot, and M. Shi, Trivial Topological Phase of CaAgP and the Topological Nodal-Line Transition in $\mathrm{CaAg}\left(\mathrm{P}_{1-x} \mathrm{As}_{x}\right)$, Phys. Rev. B 97, 161111(R) (2018).

[54] G. Cuono, C. Autieri, F. Forte, M. T. Mercaldo, A. Romano, A. Avella, and C. Noce, A Minimal Tight-Binding Model for the Quasi-One-Dimensional Superconductor $\mathrm{K}_{2} \mathrm{Cr}_{3} \mathrm{As}_{3}$, New J. Phys. 21, 063027 (2019).

[55] Y. L. Tang, Y. L. Zhu, Y. Liu, Y. J. Wang, and X. L. Ma, Giant Linear Strain Gradient with Extremely Low Elastic Energy in a Perovskite Nanostructure Array, Nat. Commun. 8, 15994 (2017).

[56] M. Goda, S. Nishino, and H. Matsuda, Inverse Anderson Transition Caused by Flatbands, Phys. Rev. Lett. 96, 126401 (2006).

[57] B. Song, C. He, S. Niu, L. Zhang, Z. Ren, X.-J. Liu, and G.-B. Jo, Observation of Nodal-Line Semimetal with Ultracold Fermions in an Optical Lattice, Nat. Phys. 15, 911 (2019).

[58] T. Ozawa, H. M. Price, A. Amo, N. Goldman, M. Hafezi, L. Lu, M. C. Rechtsman, D. Schuster, J. Simon, O. Zilberberg, and I. Carusotto, Topological Photonics, Rev. Mod. Phys. 91, 015006 (2019).

[59] J. Nissinen and G. E. Volovik, Dimensional Crossover of Effective Orbital Dynamics in Polar Distorted ${ }^{3} \mathrm{He}-\mathrm{A}$ : Transitions to Antispacetime, Phys. Rev. D 97, 025018 (2018).

[60] A. Lau, T. Hyart, C. Autieri, A. Chen, and D. I. Pikulin, Designing Three-Dimensional Flat Bands in Nodal-Line Semimetals, https://doi.org/10.5281/zenodo.3993298 (2021). 